\title{
Ethnographier le bruit en centre d'appels
}

Une analyse située de l'activité des téléopérateurs

\section{Karine Lan Hing Ting}

\section{(2) OpenEdition}

\section{Journals}

Édition électronique

URL : http://journals.openedition.org/activites/2272

DOI : 10.4000/activites.2272

ISSN : 1765-2723

Éditeur

ARPACT - Association Recherches et Pratiques sur les ACTivités

Référence électronique

Karine Lan Hing Ting, « Ethnographier le bruit en centre d'appels », Activités [En ligne], 6-2 | octobre 2009, mis en ligne le 15 octobre 2009, consulté le 01 mai 2019. URL : http://journals.openedition.org/ activites/2272 ; DOI : 10.4000/activites.2272

\section{(c) (i) (9)}

Activités est mis à disposition selon les termes de la licence Creative Commons Attribution - Pas d'Utilisation Commerciale - Pas de Modification 4.0 International. 


\title{
Ethnographier le bruit en centre d'appels : une analyse située de l'activité des téléopérateurs
}

\author{
Karine Lan Hing Ting \\ Département Sciences Economiques et Sociales - Telecom ParisTech \\ 2229 route des Crêtes, 06560 Sophia Antipolis, France \\ karine.lanhingting@telecom-paristech.fr
}

\begin{abstract}
Making an ethnographic study of noise in call centres: a situated analysis of call centre agents' work. Call centres tend to be noisy working environments, as studies in ergonomics and occupational medicine have shown. Noise in call centres can take various forms: ambient background noise - caused mainly by a large number of co-present call centre agents speaking on the phone and typing on their keyboards, high volume in the headset and instructions shouted out to the company at large. However, no employee at the call centre I have been observing seems to regard the ambient noise or the noise in the headset as a problem. Do they get used to these acoustic levels or are they unconsciously structuring the noises they perceive while at work? Making an ethnographic study of noise in call centres therefore means analysing that noise in accordance with its relevance to the activity, bearing in mind the contingencies of its production as it actually occurs, and adopting the actors' standpoint. This paper will examine two extracts - a complaint about an echo in the headset and a shouted instruction - combining a multimodal sequential analysis of speech based on transcripts of the recordings with ethnographic background data. We will see how these two sound occurrences emerge as "noises" because of the way the participants in the action categorize them.
\end{abstract}

KEYWORDS

Call center, noise, video ethnography, emic approach, accountability

\section{1.- Introduction}

Un centre d'appels est une «structure basée sur le téléphone et l'informatique qui permet une communication directe et à distance entre un interlocuteur (client, prospect, adhérent, usager...) et une personne, communément appelée téléopérateur, qui représente l'entité à l'origine du centre d'appel (entreprise, association, collectivité locale...) afin de répondre au mieux aux besoins des usagers et/ ou de développer la relation clientèle sous toutes ses formes. » (Institut des métiers de France Télécom, mars 1999). Ces téléopérateurs sont rassemblés dans un même lieu physique, un « centre » organisé en "plateaux » de tailles variables. Le centre d'appels où s'est déroulée cette observation comporte 4 plateaux, et chaque plateau accueille environ 125 personnes: 100 téléopérateurs - qui mènent leurs conversations téléphoniques respectives en même temps - 10 superviseurs, 10 responsables d'agence, deux assistants chefs de plateaux et un chef de plateau.

Qu'ils soient français, américains ou délocalisés dans les pays émergents, les centres d'appels ont la réputation d'être des environnements de travail bruyants, où, entre autres conditions de travail éprouvantes (Taylor, Baldry, Bain, \& Ellis, 2003), les téléopérateurs sont soumis à des niveaux sonores élevés. Cette « astreinte sonore », le plus souvent, résulte d'une part, de l'ambiance sonore de l'environnement de travail (qui, selon les experts, devrait être limitée à $55 \mathrm{db}$ ), et, d'autre part, du volume sonore généré par le casque d'écoute $(85 \mathrm{db})^{1}$. Les chercheurs ayant mesuré les niveaux acoustiques

1. L'institut National de Recherche et de Sécurité (Planeau, \& Robinet, 2003) consacre plusieurs documents à la sécurité au travail et aux moyens d'améliorer les conditions de travail en centre d'appels, s'agissant notamment du bruit, du 
dans les centres d'appels ont ainsi remarqué que, plus le bruit ambiant était élevé, plus les opérateurs avaient tendance à augmenter le volume de leurs casques, s'exposant ainsi à un risque auditif et à une situation de stress. Les études en ergonomie et en médecine du travail ont recommandé d'allouer 9 à $10 \mathrm{~m}^{2}$ par personne, afin de réduire le bruit ambiant mais également de limiter la gêne liée aux interférences entre locuteurs (Planeau, \& Robinet, 2003). Néanmoins, cette recommandation n'est pas toujours respectée: $25 \%$ des centres d'appels français dépasseraient les seuils admissibles de niveau sonore $($ ibid).

J'ai pu observer plusieurs types de centres d'appels, français ou délocalisés. Dans l'un d'eux, un centre d'appels interne d'un fournisseur d'accès, les téléopérateurs étaient beaucoup moins nombreux et disposaient de plus d'espace personnel de travail. Dans celui-ci, le travail des opérateurs est davantage «individuel $»^{2}$. Or, le centre décrit dans cet article peut en effet être catégorisé comme « environnement de travail bruyant ». Le travail collectif (Karsenty, \& Pavard, 1997; Benchekroun, 2000) entre superviseurs et téléopérateurs en appel y est conséquent, marqué par des pratiques coopératives (Heath, \& Luff, 1992) animées, audibles, et donc «bruyantes ». Ce centre est spécialisé en télémarketing, sous-traitant des campagnes de prospection pour divers produits et clients. Les téléopérateurs contactent des particuliers à leur domicile - appelés «prospects » - pour leur proposer une offre ou un produit commercial. Les appels s'enchaînent, menant le plus souvent à des refus juste après la présentation de l'offre que fait le téléopérateur. Ceux qui aboutissent favorablement, marqués par un intérêt clair de la part du client prospecté, sont suivis de séquences de travail collaboratif. Le deuxième extrait analysé est un travail de validation par un supérieur hiérarchique relayant un appel réussi par une téléopératrice, alors que le premier extrait survient entre deux appels de prospection de l'opératrice. Avant d'aborder l'examen de ces deux extraits, j'expose de manière générale les différentes productions sonores que j'ai pu observer dans ce centre d'appels. Cette présentation situe l'arrière-plan, à savoir que les différents « bruits » en centre d'appels surviennent couramment, intégrés de manière routinière au travail quotidien. Ensuite, je présenterai l'orientation de recherche et les méthodes de recherche, qui contribueront à mettre en perspective le choix d'analyser deux extraits en particulier.

\section{2.- Ethnographier les différents « bruits »}

Il se dégage de l'observation que les occurrences sonores (Thibaud, 1991) sont nombreuses sur le plateau et font partie du travail quotidien routinier. La présence prolongée de l'ethnographe sur le plateau lui permet d'acquérir progressivement des compétences de «membre » (Garfinkel, \& Sacks, 1970). Ainsi, l'ethnographe parvient à faire sens des différentes productions sonores, lui permettant de mieux distinguer les divers phénomènes de «bruit » sur le plateau, d'en apprécier leur variété, d'appréhender leur spécificité et leur pertinence dans l'activité, et ne plus l'envisager juste comme un «bruit». En effet, les plateaux de ce centre d'appels (Figure 1) sont souvent comparés à une ruche. La centaine de téléopérateurs sont engagés dans leurs conversations téléphoniques respectives, tapent sur leurs claviers (Zimmerman, 1992), cliquent avec la souris, tournent sur leurs chaises, bavardent avec leurs collègues entre les appels, ou collaborent verbalement avec leurs supérieurs hiérarchiques. Parfois, ils attirent l'attention des supérieurs par des sommations sonores: des claquements de doigts ou des claps de mains, qui sont audibles à plusieurs mètres de distance.

travail sur écran, de l'aménagement des postes, et des méthodes de management concernant leurs conséquences sur le bien-être des opérateurs.

2. Ce qualificatif est utilisé avec prudence étant donné que toute activité, qu'elle soit individuelle ou collective, n'en est pas moins sociale. Nos activités en tant que membres de la société sont fondamentalement interactionnelles et sont informées par le fait d'être membre de la société, et par nos relations avec les autres (Francis, \& Hester, 2004). D'ailleurs, il s'agit, dans le cas des centres d'appels, d'une activité de relation de service, qui est accomplie en interaction avec le client et l'outil informatique (Licoppe, \& Relieu, 2005). 


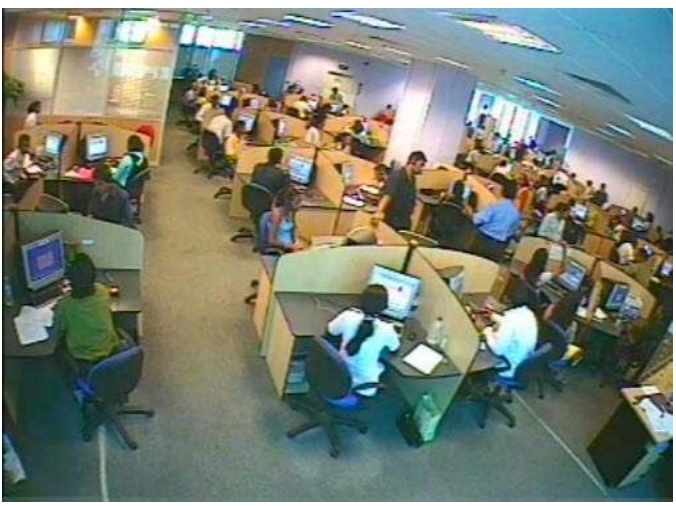

Figure 1: Vue d'ensemble du plateau

Figure 1: General view of one floor of the call centre

Certains supérieurs ou responsables d'agence se déplacent sur le plateau, pour constituer des aides volantes quand ils sont appelés par ces sommations. Le « soufflage »- où le superviseur dicte les répliques à un téléopérateur (Figure 2) dont il suit la conversation téléphonique pour l'aider à conclure une vente - est souvent crié à distance par le superviseur qui se trouve à son poste.

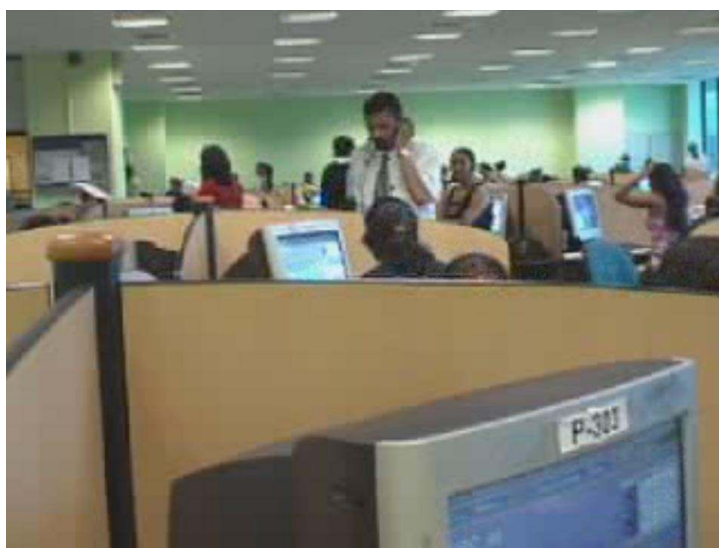

Figure 2: Le superviseur, debout, prêt à souffler au téléopérateur des répliques depuis son poste

Figure 2 : The supervisor, standing, gets ready for prompting from his desk.

Ce que je qualifie de «faire du bruit volontairement » survient également, à des moments spécifiques. Quand un téléopérateur a réussi un rendez-vous ou une vente, il est courant que l'ensemble de son équipe, une dizaine de participants l'applaudissent à l'initiative du superviseur. De même, la réunion d'équipe du matin est l'occasion de faire le point sur les résultats obtenus la veille. S'applaudir pendant la réunion d'équipe et pousser des cris de joie constitue autant une exultation qu'une motivation d'équipe en entamant la journée de travail.

Comprendre les diverses occurrences ou phénomènes de «bruit » implique d'étudier le phénomène à partir de ce qui est visible et descriptible ${ }^{3}$ comme étant un « bruit » pour les participants dans un cours d'action, et démontrable par l'analyse à partir d'orientations concrètes de la part des participants. Le mode d'enquête réflexif (Lynch, 2000) appliqué à la démarche ethnographique veut que les observations effectuées en centre d'appels ne sont pas les produits de techniques sociologiques spécialisées, mais la mobilisation d'un raisonnement pratique de sens commun (Garfinkel, 1967). En cela, la distinction classique en ergonomie entre « bruit » comme facteur de nuisance, et le « si-

3. L'accountability (Garfinkel, 1967) - la descriptibilité et le caractère reconnaissable des événements du monde social constitue l'un des concepts clé en ethnométhodologie. Voir les travaux de Salembier et Zouinar (2004) qui ont précisé la notion de «descriptibilité » dans les recherches menées sur la coopération, en la mettant en perspective par rapport à la notion de « contexte partagé » mobilisée en ergonomie. 
gnal » qui constitue une ressource (Ombredane, \& Faverge, 1955) est utile à cette analyse fine menée sur le terrain (en situation réelle de travail). Proche de ma démarche, Jean-Paul Thibaud (1991) a ethnographié notamment les pratiques communicationnelles d'ouvriers en bâtiment sur le chantier, avec et par les sons en action, en se coordonnant par l'utilisation des sons propres à ce métier. Les sons ne sont pas qu'un épiphénomène ou une conséquence secondaire de l'activité. L'environnement acoustique est une caractéristique essentielle de l'action, indissociable de l'activité. Celui-ci n'est pas donné a priori, « déjà là », mais est le produit, l'expression et la condition des pratiques sociales. La problématique est alors, non pas l'accompagnement acoustique des activités sociales mais l'accomplissement pratique de l'environnement acoustique (Thibaud, 1998).

L'on verra dans l'analyse des deux extraits et de l'accomplissement des « occurrences sonores » (ibid) et des «bruits » de quelle manière la démarcation entre les deux n'est pas donnée a priori, mais émerge en situation. Ce sont les participants qui les catégorisent comme ressource pour la coopération, ou comme entrave à l'activité en cours comme élément perturbateur qui gêne la communication téléphonique avec le client ou l'interaction collaborative sur le plateau (Kryter, 1985) ou qui représente une astreinte sonore. Il s'agira de prendre en compte le traitement des sons par les participants eux-mêmes: de leur point de vue et dans la logique organisationnelle de leur environnement de travail, et dans l'accomplissement de leur activité de travail.

L'ethnographie a toujours constitué une méthode de choix en anthropologie sociale et en ethnologie et est également l'une des méthodes couramment utilisée par les ergonomes (Blanquet, \& Poupa, 2007), s'agissant des études sur les centres d'appels, alors que la recherche sociologique a eu tendance à prioriser des méthodes de sondages et d'analyses statistiques. Depuis une vingtaine d'années, un renversement méthodologique tend à s'opérer dans les sciences sociales: l'ethnographie gagne en influence pour devenir une approche d'enquête populaire. La raison principale de ce tournant vers l'ethnographie semblerait être la reconnaissance de l'importance de la subjectivité dans la vie sociale (Francis, \& Hester, 2004, p. 22), avec l'adoption d'un point de vue émique (Pike, 1967; Winkin, 1981) ou ce que les sociologues interactionnistes de Chicago ont appelé « le point de vue de l'acteur ». Envisager les personnes en tant qu' «acteurs » en situation, détenant un ensemble de compétences et de pratiques partagées, basé sur l'expérience de travail avec les autres, nécessite de trouver de nouveaux moyens pour comprendre la relation entre les personnes, la technologie, les exigences de travail et les contraintes organisationnelles (Bannon, 1991). L'ethnographie est alors la méthode privilégiée pour aider à la conception des lieux de travail technologisés, tels que les « centres de coordination » (Suchman, 1997) dont font partie les centres d'appels. Point de rencontre entre les sciences sociales et informatiques, le courant du Computer Supported Cooperative Work préconise la conception de nouvelles technologies favorisant les pratiques collaboratives des équipes. Le CSCW a été largement inspiré par l'ethnographie ethnométhodologique dans le sillage des travaux pionniers menés par Lucy Suchman (1987). Le caractère contextuel et non théorique de l'ethnométhodologie - dont l'agenda rigoureusement descriptif force à produire des descriptions riches du travail-en-contexte (Shapiro, 1994) - s'est révélé être d'une grande valeur pour les designers (Kensing, \& Simonsen, 1997). Accédant au détail concret du travail accompli en situation, les designers peuvent alors saisir « ce qui se passe réellement », « ce qui pose vraiment un problème » dans le cours d'une activité de travail, et donc peuvent imaginer quels outils pourraient être conçus pour aider les acteurs à résoudre leurs problèmes (Hughes, Randall, \& Shapiro, 1992). La conception de systèmes collaboratifs porte un intérêt central pour le travail, l'accomplissement de procédures formelles et de la routine organisationnelle au vu des contingences quotidiennes du travail; ce que l'ethnographe apporte au design et à la conception, c'est la connaissance des pratiques de travail coopératives à travers lesquelles le travail est organisé par les participants, qu'ils soient en coprésence ou distribués à travers l'espace et le temps (Crabtree, 2003). S'éloignant des règles et des plans comme structures de contrôle de l'action qui ne déterminent ni l'accomplissement des activités de travail ni la manière dont la coordination est accomplie, cet article envisage l'analyse des pratiques de travail dans les centres d'appels en tant que «centre de coordination » (Suchman, 1997), auxquels des chercheurs s'inscrivant dans le courant des Workplace Studies (Heath, Knoblauch, \& Luff, 2000 ; Heath, \& Luff, 
2000) se sont intéressés (Zimmerman, 1992; Whalen, 1995).

\section{3.- Méthodes et orientations de recherche}

La démarche ethnographique (Crabtree, 2003) et l'approche naturaliste de la « branche vidéo de l'analyse de conversation » (ten Have, 1999), toutes deux d'inspiration ethnométhodologique, impliquent d'aborder le bruit selon l'intérêt et les contingences dans l'accomplissement de l'activité, c'està-dire en termes de mise en œuvre effective (Leplat, \& Hoc, 1983) dans la réalité du travail (Béguin, 2007). L'intérêt central de l'ethnométhodologie porte sur le caractère situé et localement accompli de l'ordre social. La vie sociale étant produite « de l'intérieur » par les membres de la société, la tâche de l'ethnométhodologie est d'identifier les méthodes d'une telle production, par des études empiriques « de bas en haut» (Hester, \& Francis, 2000). Les travaux ethnométhodologiques, principalement les travaux en analyse de conversation, ont ainsi marqué leur préférence pour des transcriptions faites à partir d'enregistrements, d'abord audio, puis vidéo (Goodwin, 1981). Ces enregistrements vidéo d'activités situées dans leur cadre ordinaire sont produits comme données audiovisuelles naturellement organisées au sein d'une perspective naturaliste initiée par Harvey Sacks (Mondada, 2008). Elles peuvent ainsi capturer et sauvegarder les phénomènes survenant de manière naturellement organisée dans leur environnement quotidien et routinier, autrement dit en situation réelle de travail. L'agenda analytique de l'analyse de conversation est de décrire les structures organisationnelles de la conduite, qui mobilisent, de manière multimodale et systématique, des ressources grammaticales, prosodiques, gestuelles et visuelles. Elles sont examinées telles que mobilisées par les participants dans l'organisation locale de leur action, sensibles aux contingences du contexte ${ }^{4}$.

Ces données d'ethnographie vidéo, collectées en multicaméra, permettent, a posteriori d'examiner certains détails multimodaux, que l'œil ou l'ouïe de l'ethnographe ne peuvent saisir entièrement en situation, ou qu'un enregistrement audio ne suffirait à aborder. De manière asynchrone à l'observation de terrain, les données donnent accès à i) la conversation téléphonique avec le prospect à laquelle l'ethnographe ne peut accéder pendant l'observation, ii) les contributions verbales et les orientations des participants vers le bruit (gestes, regard), iii) les contenus à l'écran - mouvements de souris et messages en discussion instantanée, iv) les échanges avec les participants en coprésence. De manière conjointe, les deux étapes d'une analyse séquentielle des échanges (ten Have, 1999), que sont l'enregistrement puis la transcription fine de séquences sélectionnées selon leur pertinence, permettent d'accéder, de manière répétée, au détail de l'organisation complexe de certaines activités de travail, mais également à la conversation, le parler par lequel le travail est accompli.

Avant d'aborder l'analyse des deux extraits per se, il me semble pertinent de préciser mon choix analytique de traiter ces deux extraits en détail. L'objectif de cette analyse n'est pas de produire des connaissances généralisantes et exhaustives sur le bruit en centre d'appels. Elle vise à décrire précisément l'orientation des participants dans une situation donnée, et à saisir - ce que Garfinkel (1967) nomme «thingness », «haecceity »- l'essentiel d'une situation. Un phénomène analytique n'est pas donné de manière abstraite; c'est le contexte qui fait d'une occurrence de bruit un phénomène unique, l'ordre se trouvant dans le détail. Comprendre « le whatness » d'une situation, ce qu'elle est profondément, implique d'analyser les qualités et caractéristiques qui font d'une situation donnée une situation particulière. Cela implique, en substance, d'éviter des généralisations à propos des pratiques de travail, mais d'expliquer les environnements de travail et des cours d'action spécifiques. La méthode consiste alors à analyser des cas uniques (Schegloff, 1987) d'un phénomène pertinent donné, et de montrer comment les implications productives de telles pratiques spécifiques ainsi que leur ordre, est rendu visible. Analytiquement, il s'agit, non pas d'une accumulation systématique de

4. De caractère polysémique et ubiquitaire en ergonomie (Karsenty, \& Pavard, 1997), le contexte est difficilement définissable dans les études d'inspiration ethnométhodologique (Goodwin, \& Duranti, 1992). Il n'en demeure pas moins essentiel pour comprendre le sens d'un énoncé ou d'une action (Maynard, 2003) et est aujourd'hui largement reconnu en sciences cognitives. 
connaissances, mais d'une re-spécification des objets et des produits du travail technique, comme des accomplissements locaux (Clayman, 1995). De ce fait, je focalise l'analyse sur deux extraits où le bruit émerge comme étant pertinent, en décrivant précisément les éléments distinctifs de chacun de ces phénomènes de bruit. Il s'agit de prendre ces analyses pour ce qu'elles sont: des descriptions détaillées d'un phénomène de bruit donné, et qui n'est compréhensible qu'en tenant compte du contexte dans lequel chacun d'eux émerge de manière située. En cela, cette analyse permet de comprendre précisément de quelle manière un bruit spécifique devient pertinent pour les participants dans chacun de ces deux extraits.

\section{4.- La thématisation de l'écho au casque}

Le premier extrait est une thématisation d'écho au casque, sous forme d'une plainte verbale, que la téléopératrice (TO) adresse à son superviseur (Sup, Norbert) entre deux appels. La transcription commence au moment où, pendant son appel, elle envoie un message en discussion instantanée à Ketty, une responsable d'agence, pour lui demander le nombre de ventes réussies au niveau du plateau. Elle reçoit en retour un message de Ketty, relatif au fait qu'elle est écoutée, avant de recevoir la réponse à sa question. Ces activités d'écriture-envoi-lecture des messages écrits surviennent de manière parallèle à l'activité principale de parler au téléphone avec le prospect (Pro), marqués par le rectangle. La plainte de l'écho au casque survient pendant le temps de mise en communication avec le prochain appel et est adressée à son superviseur (assis à sa droite à la même marguerite, et qu'on aperçoit de face dans les images ci-dessous, portant une chemise bleue). Elle s'adresse à lui en créole, transcrit en gras et dont la traduction figure en dessous en italique.
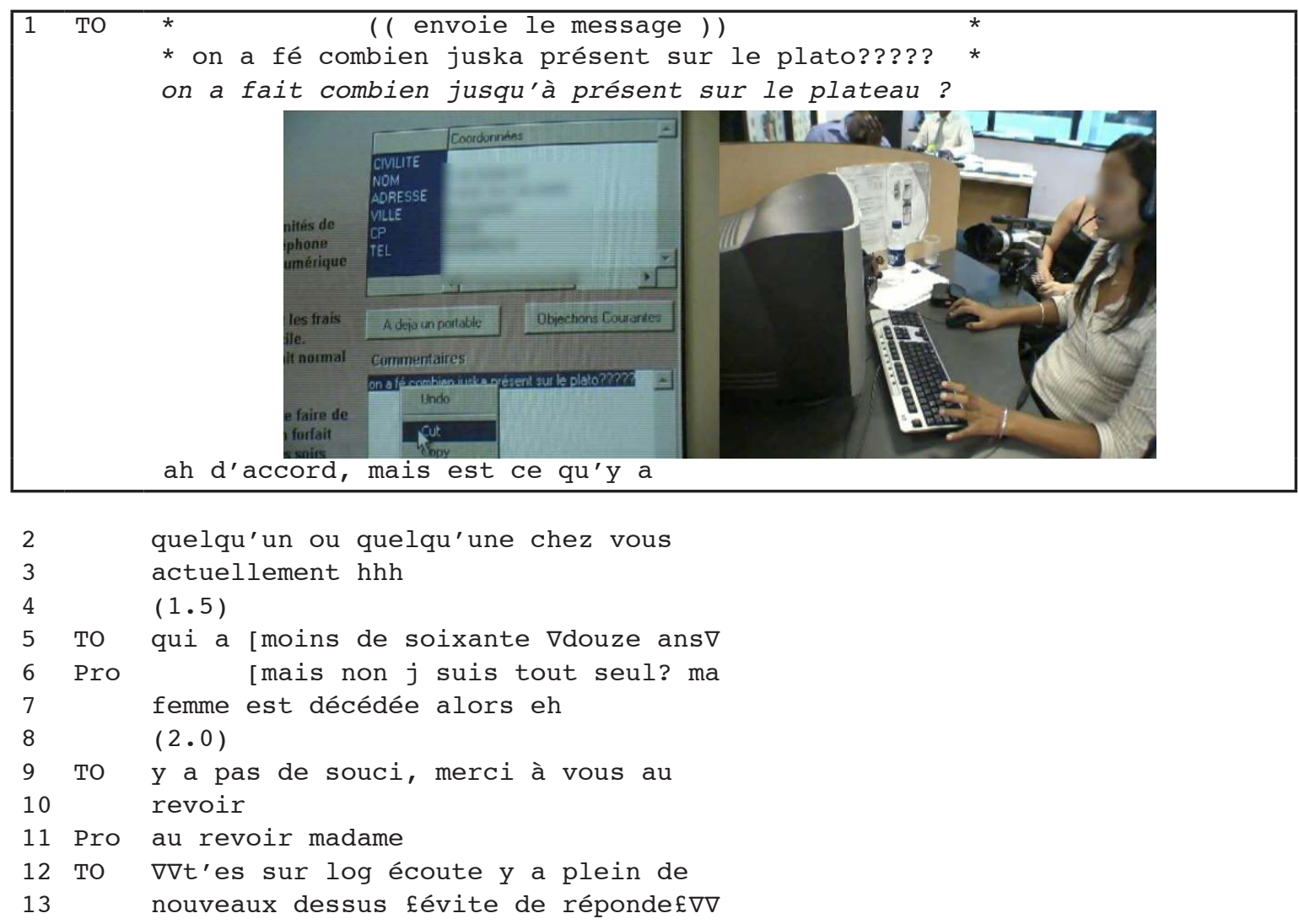
$(9.0)$

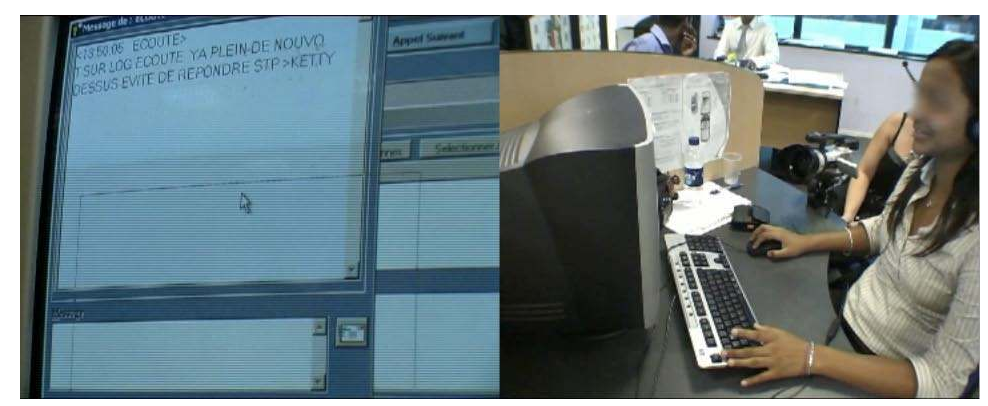

norbert? ena extra? buku eko lor mo

norbert? il y a énormément d'écho dans mon

ka?sk. dir bann nuvo evit ekut mwa

casque. dis aux nouveaux d'éviter de m'écouter

17 Sup ((regarde autour))

18 RA ((inaudible))

19 TO si ketty fek avoy mwa enn mesaz la

20 RA enn message tu?

(elle $t^{\prime} a$ ) même (envoyé) un message ?

21 TO ouais,

22 RA C'est quoi le message, (4.0) di?s

23 merci au revoir,

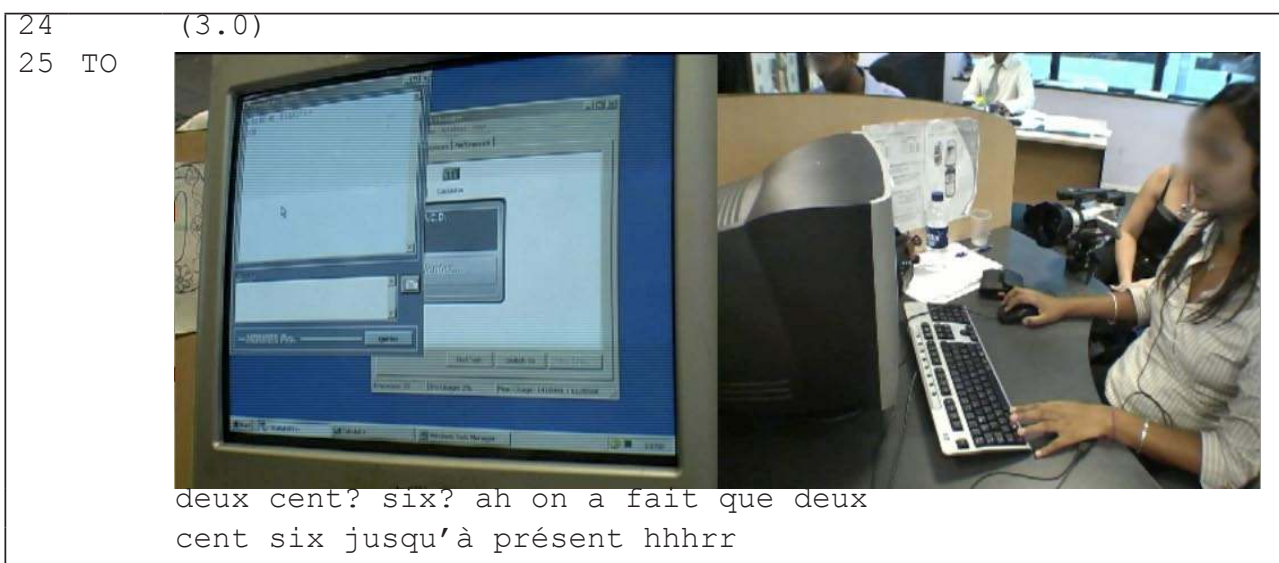

Extrait 1 : plainte et requête relatives à l'écho entendu au casque.

Extract 1: A complaint and request concerning headset echo.

La téléopératrice est toujours en appel quand elle reçoit ce message de Ketty, responsable d'agence. Ligne 4, il est visible par son silence (1.5 seconde), son froncement de sourcils et par la direction de son regard qu'elle commence à lire le message pop-up ${ }^{5}$ dès qu'il apparaît. Engagée en même temps dans son activité principale d'appel de prospection, son interaction téléphonique est quelque peu perturbée: la pause intra-tour à la ligne 4 permet plus facilement au prospect de refuser sa proposition, introduit par «mais non », ligne 6. Après la clôture de l'appel, ligne 12, elle lit à haute voix le message qu'elle vient de recevoir, avant de faire glisser la fenêtre vers le bas de l'écran (ligne 14, photo de gauche). Après neuf secondes, alors qu'elle attend la mise en connexion de son prochain appel, elle désactive son micro en le soulevant ${ }^{6}$ et énonce la plainte, en thématisant l'écho au casque,

5. Fenêtre surgissante qui s'affiche sur l'écran d'ordinateur. Cette communication quasi-synchrone partage les mêmes caractéristiques d'échanges que les logiciels de messagerie instantanée, sans toutefois reposer sur le même type de support. Nous parlerons donc de « discussion instantanée ».

6. Les appels étant composés automatiquement, il n'est pas possible de prévoir le moment où l'appelé va répondre. En désactivant le micro, les téléopérateurs ne courent pas le risque que le prospect, décrochant son téléphone, ne sur- 
en créole mauricien. La traduction littérale en français de la plainte, puis de la requête est « Norbert, il y a énormément d'écho dans mon casque. Dis aux nouveaux d'éviter de m'écouter ». En effet, la pratique routinière dans ce centre d'appels est que les nouveaux téléopérateurs, tout juste recrutés et qui sont encore en formation, «fassent de l'écoute ». Ces derniers s'asseyent à un poste et écoutent au casque les appels des téléopérateurs expérimentés ${ }^{7}$, qui sont « sur log écoute » (ligne 12), c'est-àdire, connectés de manière à pouvoir être écoutés. Plus le nombre de personnes écoutant ses appels est important, plus l'écho devient conséquent et donc potentiellement gênant pour sa conversation téléphonique. Ầ ce moment-là, en l'occurrence, «y a plein de nouveaux dessus » (ligne 12). L'écho gêne potentiellement la communication, (Kryter, 1985) tant du côté de l'opératrice que de celui du prospect à distance : il constitue donc doublement un «bruit » et est traité comme tel par le superviseur. À la thématisation de l'écho puis de la requête qui lui est adressée, le superviseur se redresse sur sa chaise et balaie le plateau du regard, à la recherche de nouveaux téléopérateurs, potentiellement sources de l'écho-bruit. Il rend ainsi visible qu'il a compris la demande et qu'il va éventuellement tenter de résoudre le problème. Bien qu'elle s'adresse nommément à Norbert, le tour de l'opératrice est entendu, compris et traité également par un responsable d'agence (autre que Ketty) - RA dans la transcription - qui se trouve à proximité. Son premier tour de parole, ligne 17, n'est pas audible. En tout cas une discussion s'ensuit entre le responsable d'agence et la téléopératrice portant sur le message envoyé par Ketty (un topic talk - Maynard, 1980), qui a précédé sa thématisation de l'écho au casque. Ligne 19, l'opératrice affirme: « Si, Ketty vient de m'envoyer un message », à quoi il répond « (elle t'a) même (envoyé) un message », qu'elle confirme. Ligne 22, il pose une question concernant le contenu exact du message. Elle n'y répond pas. On comprend par le traitement que l'opératrice fait de la question et par la direction de son regard qu'elle maintient vers ce dernier, que le responsable d'agence s'est ré-engagé dans son activité principale, auprès d'un autre téléopérateur en appel, à qui il donne des instructions, en situation (ligne 22-23).

Le fait que la conversation à propos de l'écho et du message ne soit pas poursuivie pointe vers le fait que c'est la situation de travail et l'engagement dans l'activité principale d'appels qui prime. De la même manière, l'écho n'est pas traité dans l'absolu, comme étant un bruit qui gênerait l'opératrice en termes d'astreinte physique au niveau de son audition, mais bien en termes d'entrave durant la communication téléphonique. C'est l'opératrice elle-même qui a catégorisé l'écho comme gêne à l'audibilité de la conversation téléphonique quand je lui ai posé la question, qui est survenue juste après cette séquence d'interaction transcrite. Donc, le bruit est traité par les membres du plateau uniquement quand il constitue une gêne pour l'accomplissement de l'activité d'appel. D'ailleurs, alors que l'écho dure depuis plusieurs appels ${ }^{8}$, elle ne le thématise pas avant; le message envoyé par Ketty semble séquentiellement être l'élément déclencheur. Si l'écho, par exemple, lui faisait mal aux oreilles, elle l'aurait thématisé plus tôt, et différemment. Comprendre cette séquence nécessite donc une analyse multimodale de l'interaction et de la multi-activité (Datchary \& Licoppe, 2007 ; Mondada, 2008) de chaque participant ainsi que de leurs engagements et des différents supports communicationnels. En effet, l'interaction qui survient au téléphone avec le client (lignes 1 à 11), en face à face avec les collègues ou les supérieurs hiérarchiques (lignes 15 à 22) et par écrit avec la responsable d'agence Ketty (lignes 1, 12 à 15 et 25) deviennent tour à tour, ou simultanément pertinents en termes de focalisation de l'attention de la téléopératrice. Comprendre l'organisation de l'activité de travail, l'agencement entre les multiples engagements adoptant des modes d'interaction différents, nécessite une approche interactionnelle centrée sur la séquentialité des échanges (Sacks, Schegloff, \& Jefferson, 1974) que l'enregistrement vidéo permet de préserver dans sa temporalité (ten Have, 1999; Relieu, Licoppe, \& Lan Hing Ting, à paraître), mais également une remise en contexte organisationnel de ces pratiques de communication. Ainsi, une analyse séquentielle de la conversation

prenne leur conversation en « interne ».

7. La première étape de mon ethnographie a consisté à écouter des téléopérateurs de cette manière. La liste des différents opérateurs connectés s'affichait à l'écran et il me suffisait de cliquer sur leur nom pour les écouter. Ils sont conscients qu'ils sont écoutés justement parce qu'ils entendent l'écho.

8. Je dispose de l'ensemble de l'enregistrement téléphonique avec les clients dans lesquels j’ai pu le constater. 
ne permettrait pas de comprendre tous les aspects de cette séquence de thématisation au casque. Il paraît essentiel que l'analyste mobilise la caractérisation que l'opératrice elle-même fait de l'écho au casque pendant le court « entretien » spontané qui a suivi cette séquence (l'écho gêne l'audibilité de la communication téléphonique), mais également sa connaissance d'arrière-plan (Duranti, 2001; Maynard, 2003) concernant les pratiques habituelles de travail, les catégories professionnelles des participants $^{9}$, les règles ${ }^{10}$. Une ethnographie de terrain relativement longue et approfondie, combinant la prise de notes, les discussions informelles avec les agents pour comprendre leur activité, et les enregistrements audiovisuels, permettent d'intégrer toutes les dimensions contextuelles pertinentes à l'activité dans l'analyse. En même temps, il est clair que le contexte ne doit pas constituer un cadre déterminant l'interaction. Il s'agit alors d'interpréter et de comprendre un tour de parole thématisant le bruit, ou une orientation, qu'elle soit interactionnelle, corporelle ou gestuelle, de manière située, dans le contexte de l'accomplissement de l'activité particulière de travail (Suchman, 1987).

De ce fait, l'écho au casque qu'entend l'opératrice - et vers lequel, ni l'opératrice elle-même, ni son superviseur, ni le responsable d'agence, ne s'orientent comme représentant une astreinte sonore - ne devrait pas non plus être traité par l'analyste simplement comme un «bruit». Plus qu'un simple bruit, l'écho est relatif à l'activité en cours. En cela, l'écho constitue une « information acoustique » (Thibaud, 1998), comme le son émis par les touches d'un clavier pendant son utilisation. En effet, l'écho est produit suite à la double écoute, et constitue pour l'opératrice un indice sonore qui l'informe du fait que ses appels sont écoutés par les nouveaux. Cet écho prend sens dans le contexte des procédures habituelles de travail, et constitue une ressource informationnelle pour l'opératrice. Elle s'oriente, dans sa requête, vers ce qu'implique l'écho en termes de double écoute, pas vers l'écho lui-même. Du point de vue des participants, l'écho au casque fait donc partie de l'organisation du travail de plateau et n'est pas simplement dû à une défaillance technique ou à un mauvais réglage. Néanmoins, il serait possible d'imaginer, d'un point de vue ergonomique, une amélioration du système de double écoute qui ne produirait pas d'écho gênant pour les conversations, mais qui toutefois indiquerait aux opérateurs qu'ils sont écoutés.

\section{5.- Le traitement gestuel de l'instruction-bruit}

Dans ce deuxième extrait, l'on s'intéressera à la manière dont un parler de travail, à savoir une instruction pluri-adressée produite par le chef de plateau, est traitée, de manière incarnée par le participant en action comme un bruit. Cette séquence consiste d'une étape de validation. La validation, par un responsable d'agence (appelé aussi « responsable de livraisons ») ou, éventuellement, par un superviseur comme c'est le cas ici, suit l'étape de prospection et de prise de rendez-vous, effectuée par la téléopératrice. Après avoir expliqué au prospect les conditions de l'offre, elle recueille des « informations client », telles que l'adresse, et fixe un rendez-vous pour qu'un commercial se déplace le lendemain à son domicile. Ensuite, elle passe son micro-casque à un responsable d'agence ou un superviseur pendant que la prospecte reste en ligne; celui-ci valide l'appel en confirmant que la prospecte a bien compris toutes les conditions de l'offre et du rendez-vous et lui communique également les pièces justificatives qui seront nécessaires pour établir son contrat. Ce dernier (Sup) est en communication avec la prospecte (Pro) quand le chef de plateau (CDP) annonce la pause. L'annonce instructive est lancée à la cantonade pour être entendue par l'ensemble des membres du plateau (Lan Hing Ting, \& Pentimalli, sous presse).

Pour faciliter la lecture de la transcription, il me semble pertinent de préciser les raisons pour lesquelles la transcription prend la forme de deux colonnes distinctes. La première est que la conversation téléphonique engageant le superviseur et son interlocutrice, et les échanges entendus sur le plateau émergent analytiquement comme relevant de cadres de participation (Goffman, 1974) distincts. La

9. Savoir que Ketty est une responsable d'agence qui a accès aux détails de connexion des téléopérateurs.

10. Les téléopérateurs ne peuvent communiquer par discussion instantanée qu'avec des supérieurs hiérarchiques et pas entre eux. 
prise de vue s'est focalisée sur le poste de travail où se trouve le superviseur en action, et par conséquent, les autres contributions semblent relever d'un arrière-plan sonore. Du point de vue de la cliente et en termes de conversation téléphonique distante, l'activité d'appel et l'activité de plateau ne peuvent être analysées sur un même niveau. Donc, l'on pourrait considérer que la transcription dans la colonne «Appel » traite les éléments de la conversation potentiellement pertinents pour la cliente à distance à l'avant-scène (Goffman, 1973) de la relation client (Frenkel, Korczynski, \& Tam, 1999). La colonne «Plateau » concerne les « coulisses », l'organisation de travail de plateau de manière plus globale, à laquelle, idéalement, la cliente n'a pas accès. La deuxième raison est que, $a$ priori, les deux activités ne sont pas forcément liées, même du point de vue des acteurs présents sur le plateau. Tout transcrire dans une même colonne avec des chevauchements alors qu'il s'agirait en fait de parlers parallèles n'aurait probablement pas été approprié méthodologiquement. Néanmoins, en étant mises côte à côte, avec le début de chaque partie de tour sur la même ligne pour signifier qu'elles commencent en même temps, la temporalité de l'appel comme de l'activité de plateau est préservée. La dernière raison, qui rejoint la deuxième, est d'ordre pratique, pour des motifs de compréhension et de lisibilité de la transcription.

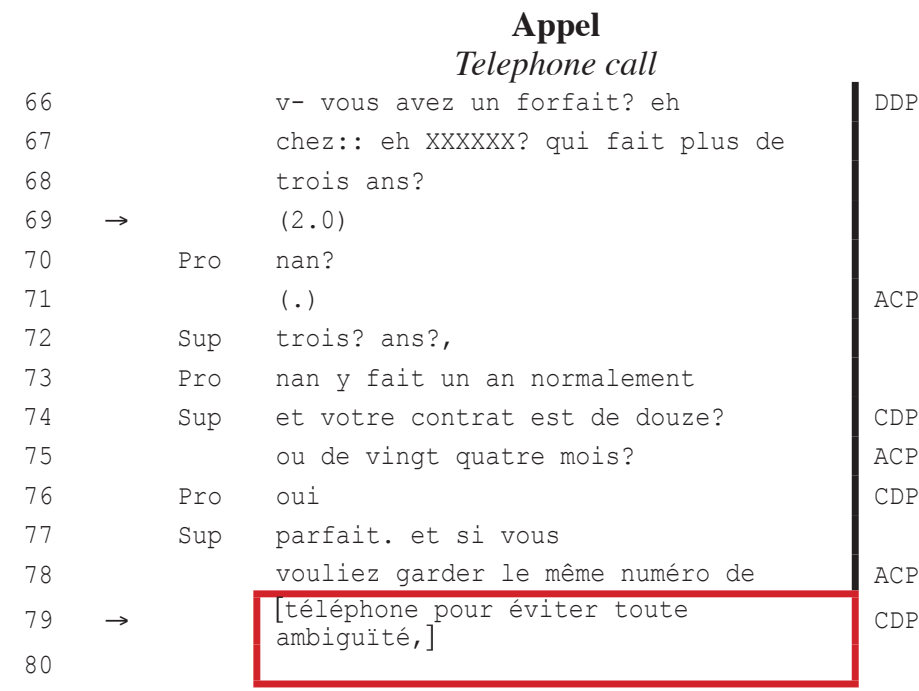

((Sup protège le micro de ses mains en entonnoir))

81 en cas d'acceptation de votre par:t

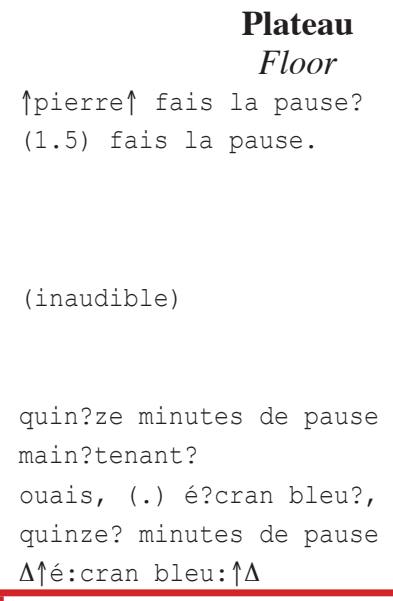

Plateau Floor

$\uparrow$ pierre^ fais la pause? (1.5) fais la pause.

(inaudible)

quin?ze minutes de pause main?tenant?

ouais, (.) é?cran bleu?, quinze? minutes de pause $\Delta \uparrow$ é:cran bleu: $\uparrow \Delta$

[ $\Delta \Delta Q \mathrm{QUIN}: \mathrm{ZE}$ MINUTES DE PAU: :SE $\uparrow \Delta \Delta]$
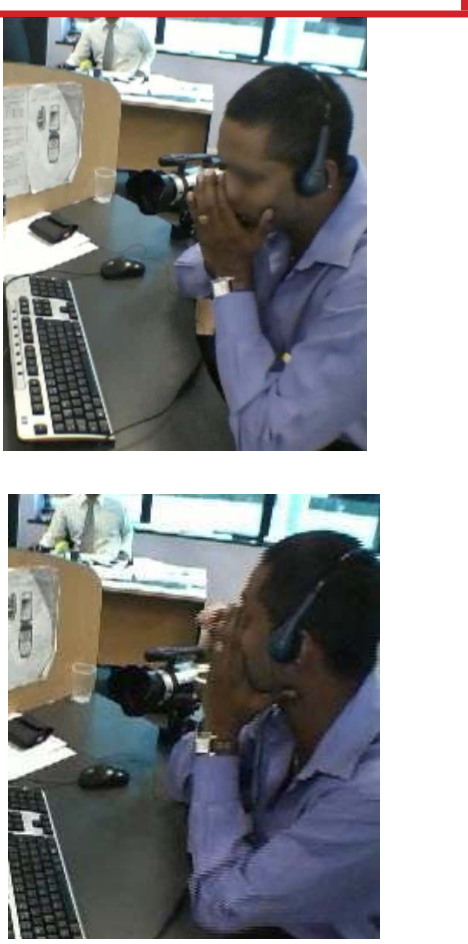
( Sup commence à enlever ses mains mais maintient son regard en direction du chef de plateau))
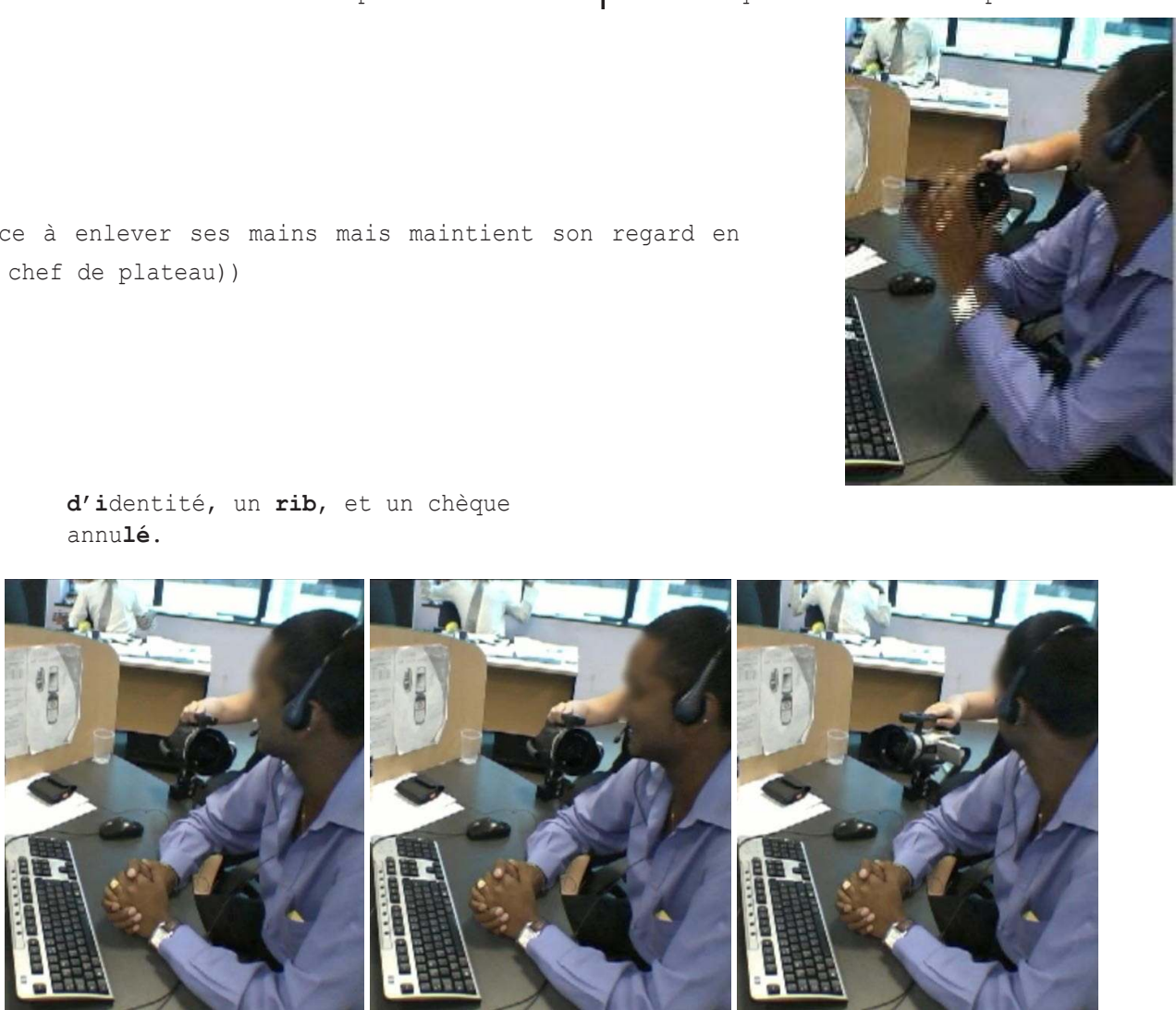

((Sup tourne la tête vers CDP, ramène sa tête en face et regarde à nouveau en direction de Pierre, à chaque Unité de Construction de Tour))

Extrait 2 : l'instruction verbale traitée comme un bruit ${ }^{11}$

\section{Extract 2: Verbal instructions handled as noise}

Sans prétendre remplacer les données originales, la transcription établie ici tente de restituer au mieux la multimodalité et la diversité des « sons » ou des «bruits » perceptibles sur le plateau, et surtout l'orientation corporelle du superviseur. Ce choix de transcription (Jefferson, 2004), pour les motifs expliqués plus haut, tend donc, dans un premier temps, à distinguer les deux activités. Néanmoins, ces deux « espaces » interactionnels se recoupent à l'annonce de la pause criée par le chef de plateau (CDP). Cette instruction « $\uparrow$ pierre $\uparrow$ fais la pause?», qui vient d'abord du directeur de production (DDP) (lignes 66 et 67, colonne plateau) est ensuite transmise par le chef de plateau à son assistant. Après une négociation entre le chef de plateau et l'assistant chef de plateau (ACP) entre les lignes 74-77, et une confirmation par le chef de plateau « ouais, (.) é?cran bleu?, quinze? minutes de pause» (lignes 76 et 77, colonne plateau), l'assistant chef de plateau (ACP) annonce « $\Delta \uparrow e ́: c r a n$ bleu: $\uparrow \Delta$ » (ligne 78). Cette annonce de l'assistant est complétée par le chef de plateau de manière sonore et ostentatoire : «[ $\Delta \Delta$ QUIN:ZE MINUTES DE PAU::SE $\uparrow \Delta \Delta]$ » (lignes 81 et 82, colonne 2). En effet, l'écran bleu se réfère à l'action de se déconnecter de son poste de travail, et est complété par l'annonce de la raison de la déconnexion - la pause - ainsi que sa durée. La transcription tente de restituer ces phénomènes vocaux: « $\Delta$ » pour le volume, les majuscules pour le fait de crier à la cantonade, et « $\uparrow$ » pour l'intonation montante.

Cette annonce à la cantonade, pluri-adressée de manière à être entendue par l'ensemble du plateau, constitue pour la plupart des membres sur le plateau, une information pertinente. Par contre, elle est traitée comme étant un bruit par le superviseur en action. Par un geste incarné des mains en forme

11. Dans cette transcription, les mots ou parties de mots en gras correspondent temporellement à l'image en dessous. 
d'entonnoir, il protège le micro de ses deux mains dans une tentative d'isoler la conversation téléphonique distante des éléments sonores provenant de son environnement proximal de travail. Il garde cette position corporelle recroquevillée, les mains en entonnoir des lignes 79 à 83 pendant qu'il poursuit sa conversation avec la cliente. Entre-temps, il regarde le chef de plateau de manière soutenue (lignes 80 à 84) pour rendre sa gêne visible, et n'enlève ses mains qu'une fois qu'il s'est assuré que le chef de plateau a observé sa gêne et que ce dernier ne produira pas d'autre annonce de ce type. Son geste est à la fois un geste pratique (empêcher que la cliente n'entende) et communicationnel (visible pour le chef de plateau). Après avoir enlevé ses mains, il maintient son regard vers le chef de plateau, dans un body torque (Schegloff, 1998), un instant de plus, en souriant. Le body torque, selon la définition de Schegloff, comme la tête désaxée par rapport au tronc, marque une orientation corporelle spécifique et un engagement autre que l'engagement principal de la partie inférieure du corps. Ce n'est qu'après quelques secondes qu'il ramène sa tête en direction de l'écran d'ordinateur, avant de jeter un dernier coup d'œil en direction du chef de plateau, et de reprendre son activité sur ordinateur: cliquer et ainsi valider l'appel avant de se déconnecter.

Par son geste incarné des mains, et le maintien du regard en direction du chef de plateau, qu'il a volontairement rendu visible pour ce dernier, le superviseur s'oriente donc vers cette annonce du chef de plateau comme étant un bruit. Nous voyons sur la vidéo ainsi que sur les captures d'images faites de ces vidéos et qui sont présentées dans cet article sous forme de photos insérées dans la transcription, que les deux participants sont placés à proximité. Le volume de cette annonce criée à la cantonade pour l'ensemble du plateau est beaucoup plus important pour ceux qui se trouvent physiquement proches du bureau du chef de plateau, comme c'est le cas pour ce superviseur. Même s'ils entendent également l'annonce du chef de plateau, la gêne sonore est plus acceptable pour les membres du plateau situés au fond de la salle. Néanmoins, malgré ce traitement de l'annonce comme bruit, le superviseur ne traite pas ce bruit en termes de volume sonore nuisible physiquement. Par exemple, il ne se bouche pas les oreilles, comme le font typiquement les personnes en entendant des pétards (Féraud, sous presse), une sonnerie d'alarme ou un marteau-piqueur. Le fait qu'il protège le micro est révélateur du fait que le bruit n'est pas pris en compte en termes d'astreinte sonore physiologique.

Cette annonce est perçue et traitée par le superviseur comme un «bruit » essentiellement en termes de contenu sémantique. « 15 minutes de pause » énoncée à ce niveau sonore est potentiellement audible par la correspondante à distance qui comprend et identifie l'annonce comme étant un parler de travail (Drew, \& Heritage, 1992). Outre les questions de face selon lesquelles annoncer la pause en criant de cette manière dans un lieu de travail serait impoli, comme le fait de se parler d'une distante trop grande qu'il ne convient selon les normes en vigueur (Goffman, 1973), c'est la « reconnaissabilité » de cette annonce à distance que le superviseur traite comme gênante pour sa conversation téléphonique en cours. Cet aspect identifiable de l'annonce-instruction s'oppose ainsi aux nombreuses sommations produites par différents téléopérateurs autour du superviseur. En effet, cinq autres sommations (lignes 21,30,34, 38 et 42) par des claps de mains ou des claquements de doigts suivent celles de l'opératrice (aux lignes 4 et 14). La sommation la plus sonore, la quatrième (ligne 38), est celle produite par le chef de plateau qui relaie celle des téléopérateurs, tout en l'amplifiant (Lan Hing Ting, \& Pentimalli, sous presse). Cette sommation attire plus particulièrement l'attention du superviseur, qui après avoir balayé le plateau du regard, tourne la tête vers le chef de plateau de manière plus marquée.

\begin{tabular}{|c|c|c|c|c|}
\hline & & $\begin{array}{c}\text { Appel } \\
\text { Telephon Call }\end{array}$ & \multirow[b]{2}{*}{$\mathrm{X}$} & $\begin{array}{c}\text { Plateau } \\
\text { Floor }\end{array}$ \\
\hline 26 & Pro & oui & & $£ \bullet H H H h £$ \\
\hline 27 & Sup & madame XXXX ? & & \\
\hline 28 & & oui: enchanté? >madame, je me & & \\
\hline 29 & $\rightarrow$ & présente:< ((tourne la tête) ) & & \\
\hline 30 & & eh je suis joël calis ((ramène la tête)) & $\mathrm{X}$ & ((2 claps des mains)) \\
\hline 31 & & responsable des livraisons? & & \\
\hline
\end{tabular}



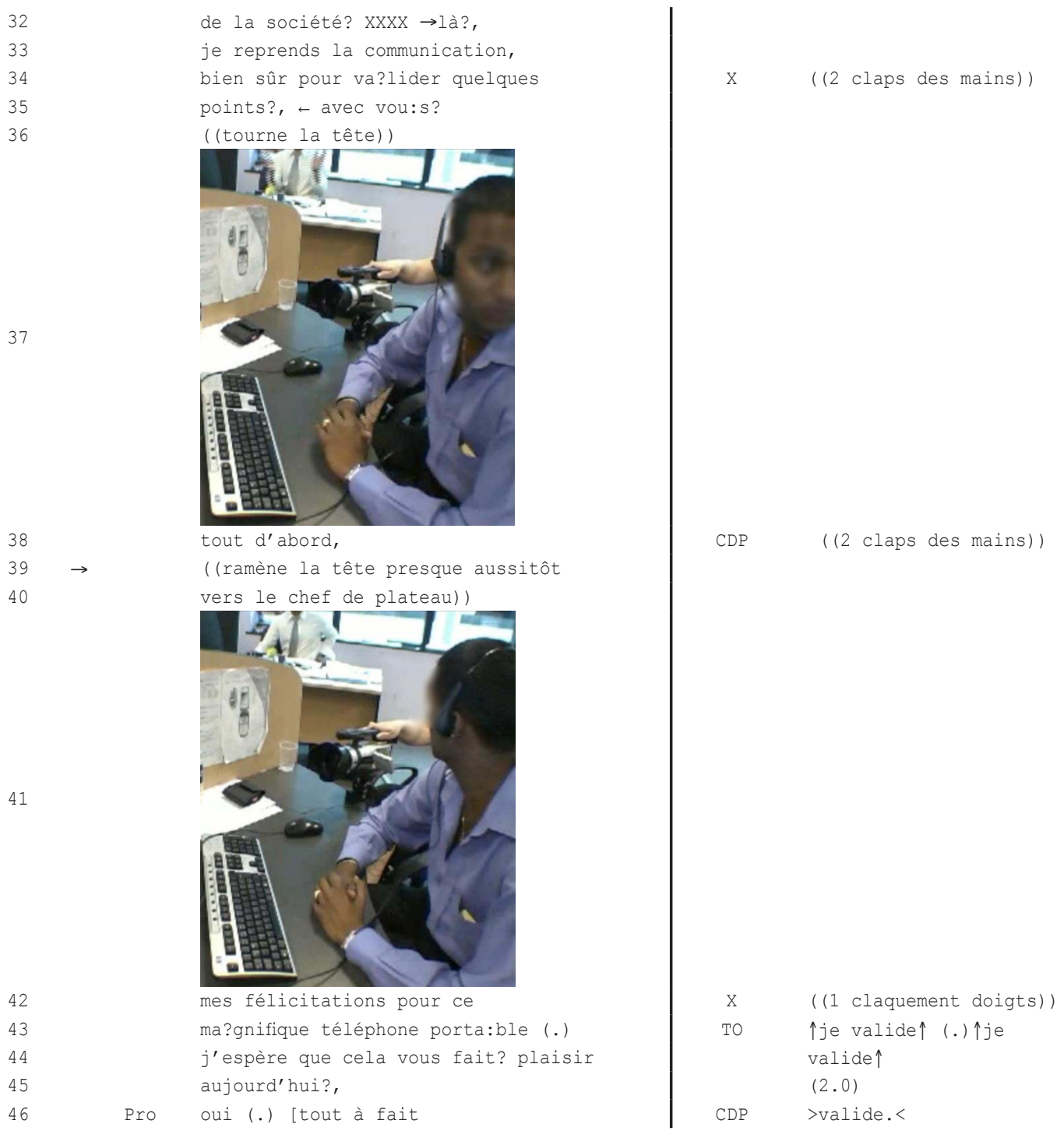

Extrait $3:$ l'instruction verbale traitée comme un bruit Extract 3: Verbal instructions handled as noise

Ces sommations successives, et précisément celle relayée par le chef de plateau, ont probablement une intensité sonore aussi grande que l'annonce-instruction, mais contrairement à cette dernière, les sommations ne sont pas identifiables et compréhensibles par la prospecte. De ce fait, les sommations ne sont pas gênantes pour le superviseur qui ne les traite pas comme des bruits, mais au contraire comme de réelles sommations, des moyens d'attirer l'attention et appeler des supérieurs hiérarchiques. Des études ont montré que ce sont le parler et les activités humaines (distractive speech noise) qui déconcentrent le plus en open office (Helenius, Keskinen, Haapakangas, \& Hongisto, 2007). Distinguant quatre types de bruit - bruit blanc de type 2, bruit de circulation routière, parole non pertinente dénuée de sens et enfin parole pertinente significative - Zucchi et Gamberini (2007 : 209) prouvent que ce sont les formes verbales de bruit, et plus particulièrement la dernière qui constitue une gêne en termes d'activités cognitives. Il est possible d'en déduire que dans les activités de centre d'appels également, caractérisés par une écologie en open office, c'est la parole pertinente significative, qui est compréhensible et audible, qui constitue la forme de « bruit » la plus perturbante.

L'annonce-instruction est également traitée comme un bruit étant donné qu'il s'agit ici de deux environnements interactionnels distincts, restitués dans la transcription dans deux colonnes: à droite, l'environnement spatial et sonore du plateau où les membres en coprésence collaborent, potentielle- 
ment par des pratiques communicatives bruyantes; et à gauche, l'espace interactionnel de la conversation au téléphone à distance, où la conversation est médiée par le Couplage Téléphonie-Informatique. Au sein de cette distinction, l'espace de travail pourrait être considéré comme les «coulisses » et l'espace de la conversation comme étant «l'avant-scène », comme le qualifierait Goffman (1973). De ce fait, quand un parler interne de travail effectué en coprésence sur le plateau s'immisce dans la conversation à distance au téléphone à travers les caractéristiques socio-matérielles du casque, les deux espaces se mélangent alors qu'idéalement les éléments de coulisses sont supposés y rester. Ils deviennent donc potentiellement des éléments perturbateurs de la conversation et sont catégorisés comme « bruits », comme c'est le cas pour ce superviseur.

Le placement séquentiel de cette annonce dans le cours de la conversation contribue également au fait que le superviseur la perçoive comme gênante. La fin de l'appel doit donner aux deux interlocuteurs le sentiment qu'ils se quittent alors qu'il n'y a plus rien à se dire après une amorce appropriée de la séquence de clôture (Schegloff, \& Sacks, 1973). Or, l'annonce de la pause pourrait donner le sentiment à la prospecte que l'agent va se débarrasser hâtivement des explications afin de pouvoir partir en pause au plus vite, et donc qu'elle ne serait pas prise au sérieux. D'un point de vue interactionnel, ce genre de perturbation pourrait être nuisible à la relation commerciale. De plus, à ce moment de l'appel, le superviseur est en train de confirmer l'intérêt de la prospecte pour l'offre; la perturbation du bruit survient juste avant qu'il ne lui communique la liste des pièces qu'elle devra fournir au commercial qui va se déplacer à son domicile. Il s'agit donc d'un moment important de l'appel en termes d'activités à accomplir. Si la prospecte ne comprend pas la liste des pièces à fournir au commercial le lendemain, soit le commercial va perdre du temps, soit le contrat ne pourra être établi, dans lequel cas, cette vente sera perdue. Quand une vente est perdue, non seulement la téléopératrice ne touche pas de prime, mais en plus, un commercial se sera déplacé « pour rien ». Dans tous les cas, c'est du temps de travail et de l'argent perdu.

Dans un tel espace séquentiel de l'appel - dans lequel elle s'immisce alors qu'elle devrait rester en arrière-plan - l'instruction sonore est problématique et devient un bruit. Ce bruit est traité comme tel par le superviseur de manière visible de par son orientation gestuelle de protéger le micro de ses mains, et par son orientation visuelle vers le chef de plateau. Néanmoins, cette instruction constitue principalement, du point de vue des autres membres du plateau, une information pertinente, en termes d'organisation de l'activité. Elle participe également d'une dynamique joyeuse de partir en pause, vers lequel le superviseur s'oriente également: il sourit pendant qu'il regarde le chef de plateau.

\section{6.- Conclusion}

La plupart des recherches portant sur les centres d'appels, qu'elles soient sociologiques utilisant des analyses statistiques (Taylor et al., 2003), ou de sécurité mesurant les niveaux sonores (Planeau, \& Robinet, 2003), s'accordent à démontrer que le centre d'appels est un lieu de travail bruyant. Néanmoins la communication interpersonnelle n'y est pas forcément difficile (Kryter, 1985) et les astreintes sonores inévitables. Cet article a examiné l'organisation du parler et du cours d'action d'un point de vue émique (Pike, 1967; Winkin, 1981) et de manière située, pour interroger si l'environnement bruyant est pertinent pour les participants et de quelle manière sa pertinence serait rendue visible et démontrable par et pour les interactants. Les occurrences sonores dans les deux extraits analysés, qu'il s'agisse de la thématisation verbale de l'écho au casque (extrait 1) ou de l'instruction pluri-adressée à laquelle le superviseur s'oriente par le geste des mains en entonnoir (extrait 2), sont de manière visible pour les autres participants et démontrable pour l'analyse, catégorisables comme « bruit ». Le «bruit » émerge comme étant un élément perturbateur, soit parce que l'écho gêne l'audibilité de la communication téléphonique, soit parce que l'instruction peut être entendue par la prospecte à un placement séquentiel de l'appel délicat, les coulisses s'immisçant dans la conversation durant l'étape importante de validation. Aucun des deux participants, dont l'action a été décrite, ne s'oriente vers le bruit comme une astreinte sonore. Ces deux phénomènes de «bruit » ne sont pleinement compréhensibles qu'en tenant compte du contexte qui font qu'ils émergent comme «bruits ». Ils ne peuvent être généralisables pour affirmer que le centre d'appels est un environnement bruyant. 
Néanmoins, vu les orientations de gêne constatées, il serait possible d'imaginer une amélioration du système de double écoute qui ne produirait pas d'écho gênant pour les conversations, mais qui toutefois indiquerait aux opérateurs qu'ils sont écoutés. En effet, l'écoute des appels d'un opérateur entraînant un écho à son casque est un cas d'usage parfois problématique. De la même manière, à partir de mon retour de terrain, j'ai pu observer l'usage régulier de messages sous formes de fenêtres apparaissant sur chaque écran d'ordinateur, pluri-adressés à l'ensemble du plateau, pour informer des pauses ou de la fin du travail. Il serait également possible d'envisager un usage plus régulier et une « usabilité » (Nielsen, 1993) améliorée de ces outils, tout en tenant compte du fait que les annonces à la cantonade participent d'une certaine dynamique d'équipe. En effet, le superviseur s'oriente vers cette dynamique joyeuse qu'est l'annonce de la pause: il ne montre pas d'agacement, mais sourit en regardant le chef de plateau ayant produit l'instruction-bruit. Il est donc essentiel, que l'objectif analytique soit une description fine en vue de comprendre l'émergence du bruit, ou en vue d'une amélioration du travail, d'analyser ces occurrences de bruit en tenant compte de l'orientation des participants vers l'environnement matériel de son occurrence, de son contexte social, matériel, séquentiel et praxéologique. En effet, l'écho au casque est à la fois une ressource informationnelle concernant le fait qu'elle est écoutée, qu'une entrave à l'audibilité de la communication téléphonique. De même, l'instruction constitue une information pertinente pour l'ensemble du plateau, alors qu'elle constitue une gêne pour le superviseur. En tenant compte de ces différents points de vue, le centre d'appels apparaît alors comme, non pas simplement un environnement bruyant, mais un lieu de travail bruissant d'activités socialement organisées.

\begin{tabular}{|c|c|}
\hline Symbole & Description \\
\hline $\mathrm{TO}$ & Téléopératrice \\
\hline Sup & Superviseur \\
\hline Pro & Client prospect \\
\hline CDP & Chef de plateau \\
\hline $\mathrm{ACP}$ & Assistant chef de plateau \\
\hline RA & Responsable d'agence \\
\hline DDP & Directeur de production \\
\hline $\mathrm{X}$ & Locuteur non identifié \\
\hline$\Delta$ & Hausse de volume \\
\hline$\uparrow$ & Intonation montante \\
\hline MAJUSCULES & Annonces à la cantonade \\
\hline$=$ & Enchaînement rapide entre énoncés de locuteurs différents \\
\hline[ & Chevauchement d'énoncés \\
\hline hhh & Inspiration audible \\
\hline() & Pause longue chronométrée en secondes \\
\hline$(())$ & Phénomènes décrits mais non transcrits \\
\hline$(-)$ & Pause en dixièmes de seconde \\
\hline (.) & Micro pause de moins d'un dixième de seconde \\
\hline$?$ & Intonation montante moindre que $\uparrow$ \\
\hline$?$, & Intonation légèrement montante \\
\hline , & Intonation continue \\
\hline$\downarrow$ & Intonation descendante \\
\hline . & Intonation moins descendante que $\downarrow$ \\
\hline$!$ & Ton animé \\
\hline- & Interruption subite de la parole \\
\hline$><$ & Accélération du rythme de l'énoncé \\
\hline$:$ & Allongement du son ou de la syllabe \\
\hline$=$ & Emphase sur un mot ou une partie de phrase \\
\hline$\nabla$ & Baisse de volume \\
\hline 0 & Ton plus calme par rapport au reste de l'énoncé \\
\hline$\&$ & Continuation d'un même tour après un chevauchement \\
\hline $\mathrm{XXX}$ & Nom du client ou numéro censuré \\
\hline$£$ & Sourire dans la voix pendant l'énoncé \\
\hline
\end{tabular}

Tableau 1 : Index de notations de transcription et d'abréviations des catégories des participants

Table 1: Transcript notation symbols and abbreviations index 


\section{BIBLIOGRAPHIE}

Bannon, L. J. (1991). From human factors to human actors: The role of psychology and human-computer interaction studies in system design. In J. Greenbaum, \& M. Kyng (Eds.), Design at work: Cooperative design of computer systems §pp. 25-44). Hillsdale, NJ: Lawrence Erlbaum Associates.

Béguin, P. (2007). Prendre en compte l'activité de travail pour concevoir. @ ctivités, 4(2), 107-14, http:// www.activites.org/v4n2/beguin-FR.pdf

Benchekroun, T. H. (2000). Les espaces de coopération proxémique. In T. H. Benchekroun, \& A. WeillFassina (Eds.), Le travail collectif : perspectives actuelles en ergonomie (pp. 35-53). Toulouse: Octarès Editions.

Blanquet, J., \& Poupa, M. M. (2007). Fiabiliser, enrichir la relation client en contexte SAV. Actes du 42ème Congrès de la Société d'Ergonomie de Langue Francaise (Saint-Malo; 5-6-7 septembre)

Clayman, H. M. (1995). The dialectic of ethnomethodology. Semiotica, 107(1/2), 105-123.

Crabtree, A. (2003). Designing collaborative systems: A practical guide to ethnography. London: SpringerVerlag.

Datchary, C., \& Licoppe, C. (2007). La multi-activité et ses appuis : l'exemple de la «présence obstinée» des messages dans l'environnement de travail. @ctivités, 4(1), 4-29.

Drew, P. \& Heritage, J. (1992). Analyzing talk at work: an introduction. In P. Drew, \& J. Heritage (Eds.), Talk at work: interaction in institutional settings (pp. 3-65). Cambridge: Cambridge University Press.

Duranti, A. (2001). Universal and culture-specific properties of gretings. In A. Duranti (Ed.), Linguistic Anthropology: a reader (pp. 208-238). Malden, Mass: Blackwell Publishing.

Féraud, O. (sous presse). Le bruit et la lumière : une anthropologie sonore des pétards et des feux d'artifice à Naples. Ethnographiques.org, 19.

Francis, D., \& Hester, S. (2004). An Invitation to Ethnomethodology: Language, Society and Interaction. London: Sage Publications

Frenkel, S., Korczynski, M., A., S. K., \& Tam, M. (1999). On the Front Line: Organization of Work in the Information Economy. Ithaca: Cornell University Press.

Garfinkel, H. (1967). Studies in Ethnomethodology. Englewood Cliffs, Nj: Prentice Hall

Garfinkel, H., \& Sacks, H. (1970). On formal structures of practical actions. Theoretical Sociology: Perspectives and Developments, 160-193.

Goffman, E. (1973). La mise en scène de la vie quotidienne. Paris: Les Editions de Minuit.

Goodwin, C. (1981). Conversatioinal organization: interaction between speakers and hearers. New York: Academic Press.

Goodwin, C., \& Duranti, A. (1992). Rethinking context: an introduction. In A. Duranti, \& C. Goodwin (Eds.), Rethinking context: Language as an interactive phenomenon (pp. 1-42). Cambridge: Cambridge University Press.

Goffman, E. (1974). Frame analysis: An essay on the organization of experience. New York: Harper and Row.

Have, P. ten, (1999). Doing Conversation Analysis: A Practical Guide. London: SAGE.

Heath, C., \& Hindmarsh, J. (2002). Analysing interaction: Video, ethnography and situated conduct. Qualitative Research, 99-121.

Heath, C., Knoblauch, H., \& Luff, P. (2000). Technology and Social Interaction: the Emergence of 'workplace Studies'. The British Journal of Sociology, 51(2), 299-320.

Heath, C., \& Luff, P. (1992). Collaboration and control: crisis management and multimedia technology in London Underground control rooms. Journal of Computer Supported Cooperative Work, 1, 69-94.

Heath, C., \& Luff, P. (Eds.) (2000). Technology in Action. Cambridge: Cambridge University Press. 
Helenius, R., Keskinen, E., Haapakangas, A., \& Hongisto, V. (2007). Acoustic environment in finnish offices - the summary of questionnaire studies. Paper presented at the 19th International Congress on Acoustics, 2-7 September 2007, Madrid.

Hester, S. \& Francis, D. (Eds.). (2000). Local educational order: ethnomethodological studies of knowledge in action. Amsterdam: John Benjamins.

Hughes, J. A., Randall, D., \& Shapiro, D. (1992). Faltering from ethnography to design. Proceedings of the 1992 ACM Conference on Computer-supported cooperative work, 115-122.

Institut des Métiers de France Telecom, (1999). Les Centres D’appels En France : Synthèse Des Principaux Écrits. Paris: France Telecom

Jefferson, G. (2004). Glossary of transcript symbols with an introduction. In G. H. Lerner (Ed.), Conversation analysis: Studies from the first generation (pp. 13-31). Amsterdam/Philadelphia: John Benjamins.

Karsenty, L., \& Pavard, B. (1997). Différents niveaux d'analyse du contexte dans l'étude ergonomique du travail collectif. Réseaux, 85, 73-99.

Kensing, F., \& Simonsen, J. (1997). Using ethnography in contextual design. Communications of the ACM, $40(7), 82-88$.

Kryter, K. D. (1985). The effects of noise on man. New York: Academic Press.

Lan Hing Ting, K., \& Pentimalli, B. (sous presse). Le « bruit » comme ressource pour la coopération et la coordination entre téléopérateurs dans les centres d'appels. Ethnographiques.org, 19.

Leplat, J., \& Hoc, J. M. (1983). Tâche et activité dans l'analyse psychologique des situations. Cahiers de psychologie cognitive, 3(1), 49-63.

Licoppe, C., \& Relieu, M. (2005). Entre système et conversation. Une approche située de la compétence des téléopérateurs dans les services d'assistance technique. In E. Kessous, \& J.L. Metzger (Eds), Le Travail Avec Les Technologies De L'information (pp. 177-199). Paris: Hermès.

Lynch, M. (2000). Against Reflexivity as an Academic Virtue and Source of Privileged Knowledge. Theory, Culture, and Society, 17 (3), 27-56.

Maynard, D. W. (1980). Placement of topic changes in conversation. Semiotica, 30, 263-290.

Maynard, D. W. (2003). Bad news, good news: Conversational order in everyday talk and clinical settings. Chicago, Ill.; London: University of Chicago Press.

Mondada, L. (2008). Using video for a sequential and multimodal analysis of social interaction: videotaping institutional telephone calls. Forum Qualitative Sozialforschung / Forum: Qualitative Social Research, 9(3), 88 paragraphs.

Nielsen, J. (1993). Usability Engineering. Boston: Academic Press.

Ombredane, A., \& Faverge, J.-M. (1955). L'analyse du travail : facteur d'économie humaine et de productivité. Paris: PUF.

Planeau, V., \& Robinet, D. (2003). Evaluation De L'exposition Sonore Quotidienne Des Opérateurs De Centres D’appels Téléphoniques. Institut national de recherche et de sécurité NS, 231.

Pike, K. L. (1967). Language in relation to a unified theory of the structure of human behavior. The Hague: Mouton.

Relieu, M., Licoppe, C., \& Lan Hing Ting, K. (à paraître). Filmer le travail dans les centres d'appels : le cadrage vidéo et sonore comme mise à l'échelle de l'activité. Actes du Colloque Filmer le travail, Film et Travail, Aix en Provence, 21-24 novembre 2007.

Salembier, P., \& Zouinar, M. (2004). Intelligibilité mutuelle et contexte partagé. Inspirations conceptuelles et réductions technologiques. @ctivités, 1(2),64-85.

Sacks, H. (1992). Lectures on conversation (Edited by Gail Jefferson, with introduction by Emanuel A. Schegloff). Oxford, UK ; Cambridge, Mass: Blackwell. 
Sacks, H., Schegloff, E., \& Jefferson, G. (1974). A simplest systematics for the organization of turn taking for conversation. In J. N. Schenkein (Ed.), Studies in the organization of conversational interaction (pp. 7-55). New York: Academic Press.

Shapiro, D. (1994). The limits of ethnography: Combining social sciences for CSCW. Proceedings to the 1994 ACM Conference on Computer Supported Cooperative Work, pp. 417-428.

Schegloff, E. A. (1987). Analyzing single episodes of interaction: an exercise in conversation analysis. Social Psychology Quarterly, 50, 101-114.

Schegloff, E. A. (1998). Body Torque. Social Research, 65(3), 535-596.

Schegloff, E. A., \& Sacks, H. (1973). Opening up closings. Semiotica, 8, 289-327.

Shapiro, D. (2005). Participatory design: the will to succeed. Proceedings of the 4th decennial conference on Critical computing: between sense and sensibility, 29-38.

Suchman, L. (1987). Plans and situated actions: the problem of human-machine communication. Cambridge: Cambridge University Press.

Suchman, L. (1997). Centers of Coordination: a case and some themes. In L. B. Resnick, Säljö, R. Pontecorvo, C., \& Burge, B. (Eds.), Discourse, tools, and reasoning: essays on situated cognition (pp. 41-62). Berlin: Springer-Verlag.

Taylor, P., Baldry, C., Bain, P., \& Ellis, V. (2003). 'A unique working environment': health, sickness and absence mangagement in UK call centres. Work, Employment \& Society 17(3), 435-458.

Thibaud, J. P. (1998). The acoustic embodiment of social practice. Proceedings of the Conference Stockholm, Hey Listen!, pp. 17-22.

Thibaud, J. P. (1991). Temporalités sonores et interaction sociale. Architecture et Comportement / Architecture and Behaviour, 7(1), 63-74.

Whalen, J. (1995). Expert systems versus systems for experts: computer-aided dispatch as a support system in real-world environments. In P. Thomas (Ed.), The Social and Interactional Dimensions of HumanComputer Interfaces (pp. 161-183). Cambridge: Cambridge University Press.

Winkin, Y. (1981). La Nouvelle Communication. Paris: Editions du Seuil.

Zimmerman, D. H. (1992). The interactional organization of calls for emergency. In P. Drew, P., \& J. Heritage (Eds.), Talk at work: interaction in institutional settings (pp. 418-469). Cambridge: Cambridge University Press.

Zucchi, A., \& Gamberini, L. (2007). Searching for information on PDA in a naturalistic environment with or without music. PsychNology Journal, 5(2), 207-222.

\section{RÉSUMÉ}

Les centres d'appels sont considérés comme des environnements de travail bruyants, selon les études en ergonomie et en médecine du travail. Les bruits perçus sont divers : le bruit ambiant - causé principalement par les voix et l'utilisation des claviers des nombreux téléopérateurs présents sur la même plateforme - le bruit au casque, ou les annonces sonores lancées à la cantonade. Pourtant, aucun membre du plateau de centre d'appels observé ne semble s'orienter vers le bruit ambiant, ou le bruit au casque comme astreinte sonore. S'agit-il d'une habituation aux niveaux acoustiques ou d'une structuration de la perceptibilité des différentes productions sonores pendant l'activité ? De ce fait, ethnographier le bruit en centre d'appels implique de l'aborder selon sa pertinence dans l'activité et du point de vue des téléopérateurs, en tenant compte des contingences de sa production en situation de travail réel. Cet article s'intéressera à deux extraits - une plainte concernant un écho au casque et une annonce à la cantonade - en combinant une analyse séquentielle multimodale des échanges basée sur la transcription des enregistrements, à une mobilisation de connaissances contextuelles d'arrière-plan acquises par l'ethnographie. L'on verra comment ces deux occurrences sonores émergent comme «bruits » par la 
catégorisation qu'en font les participants en action.

MoTS CLÉ :

centre d'appels, bruit, ethnographie vidéo, approche émique, descriptibilité

\section{RÉFÉRENCEMENT}

Lan Hing Ting, K. (2009). Ethnographier le bruit en centre d'appels : une analyse située de l'activité des téléopérateurs. Activités, 6 (2), pp. 100-118. http://www.activites.org/v6n2/v6n2.pdf

Article soumis le 9 mars 2009 accepté pour publication le 8 septembre 2009 